\title{
Testing the nonclassicality of spacetime: what can we learn from Bell-Bose et $a l$. -Marletto-Vedral experiments?
}

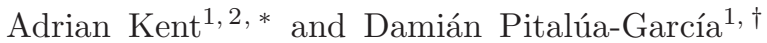 \\ ${ }^{1}$ Centre for Quantum Information and Foundations, DAMTP, \\ Centre for Mathematical Sciences, University of Cambridge, \\ Wilberforce Road, Cambridge, CB3 OWA, United Kingdom \\ ${ }^{2}$ Perimeter Institute for Theoretical Physics, 31 Caroline Street North, Waterloo, ON N2L 2Y5, Canada
}

(Dated: January 4, 2022)

\begin{abstract}
The Bose et al.-Marletto-Vedral (BMV) experiment [S. Bose et al., Phys. Rev. Lett. 119, 240401 (2017); C. Marletto and V. Vedral, Phys. Rev. Lett. 119, 240402 (2017)] aims to prove that spacetime is nonclassical by observing entanglement generated by gravity. However, local hidden variable theories (LHVTs) can simulate the entangled correlations. We propose to extend the entanglement generated by the BMV experiment to distant quantum particles in a Bell experiment. Violating a Bell inequality would rule out LHVTs, providing a stronger proof of the nonclassicality of spacetime than the BMV proposal.
\end{abstract}

\section{INTRODUCTION}

We do not yet know how to unify our two most fundamental theories of physics, quantum theory (QT) and general relativity (GR). Each is supported by overwhelming experimental evidence in its respective domain. However, they are formulated very differently. GR is an essentially classical theory in which the stress-energy tensor, the spacetime geometry and all physical quantities take well-defined values; QT predicts that distinct massenergy configurations can be in quantum superposition. One popular approach to resolving this tension is to seek a quantum theory of gravity, such as string theory [1] or loop quantum gravity [2]. It has even been claimed that there is no consistent alternative (e.g., [3 6$]$ ). However, this has also often been questioned (e.g. 7]). In particular, the argument of Ref. [4] has been refuted (e.g., [8 11]). It seems the question can only be settled empirically.

In their simplest form, semi-classical gravity models 12 15] are defined by taking

$$
G_{\mu \nu}=\kappa\left\langle\hat{T}_{\mu \nu}\right\rangle,
$$

where the left hand side is the Einstein tensor of classical spacetime, and the right hand side is the expectation value of the stress-energy tensor of quantum matter propagating in that spacetime, with $\kappa$ being a proportionality constant. If this is taken to be defined by Everettian quantum theory with purely unitary evolution, Eq. (11) is inconsistent with observation (e.g. [16]); it is also unclear that it defines a consistent theory. However, it remains possible that (10) holds in some regime (e.g. [17 20]). For example, given a version of quantum theory with explicit localized collapses, a classical gravitational field could couple to the local quantum state,

\footnotetext{
* A.P.A.Kent@damtp.cam.ac.uk

$\dagger$ D.Pitalua-Garcia@damtp.cam.ac.uk
}

which is defined by the initial conditions, evolution, and collapse events (only) in the causal past of the relevant point [11, 20, 21]. Another possibility is that quantum superpositions of sufficiently distinct energy-mass configurations are dynamically suppressed (e.g., 22 29]).

All these options are problematic. For example, a recent experiment [30] gives strong evidence against the Diósi-Penrose proposals [24 26, 28, 29] for gravitationally induced collapse and suggests that a radically new approach may be needed to pursue this idea. However, quantum gravity theories also have well known problems (see e.g. 31] for some discussion).

Recent proposals for table-top tests of quantum gravity, beginning with so-called Bose et al.-Marletto-Vedral (BMV) experiments [32, 33], aim to give strong evidence as to whether gravity is mediated by quantum information exchange. This would also give indirect evidence that spacetime is quantum 34. The essential idea of BMV's proposal is to place two adjacent mesoscopic masses in position superposition states and allow them to fall along paths such that one pair of paths is significantly closer than the others, before recombining the paths interferometrically. With appropriate masses, separations, and fall times, which it is hoped will be experimentally feasible in the foreseeable future, the Schrödinger evolution with a Newtonian potential implies that an entangled final state can be generated from a separable initial state. As the position degrees of freedom are correlated with internal degrees of freedom, this entanglement can be tested by measuring entanglement witnesses.

Refinements to the experiments have since been proposed [35], as have alternative experiments aiming to witness (non-)quantum behaviour of gravity by other methods (e.g., 29, 36 38]). There is also ongoing debate (e.g. [37, 39, 40]) over how definitively the quantum nature of gravity would be demonstrated by the generation of entanglement in a BMV experiment.

A separate issue, our focus here, is how definitively passing the type of entanglement witness test proposed by BMV would establish that entanglement had indeed 
been generated. There is clearly a logical loophole in this inference, since we know that local hidden variable theories can simulate arbitrary entangled correlations in any experiment - such as the BMV proposals - that does not involve measurements in spacelike separated wings. Of course, Bell experiments give very strong - even if not yet loophole-free [41, 42] - evidence against local hidden variable theory explanations for correlations between measurements on entangled matter. However, Bell experiments to date have not tested states in which (according to quantum gravity intuitions) entanglement is generated by gravitational interactions. As already noted, there is some motivation for considering models in which classical degrees of freedom associated with gravity couple to, and might indeed contain complete information about, the local quantum state. In principle, this would allow classical simulation of the correlations that a quantum analysis would ascribe to gravitationally generated entanglement.

We are not aware of any convincing model that would reproduce the correct correlations for all the entangled states that could (on a quantum analysis) be generated by varying the experimental parameters. Still, BMV experiments aim to establish a fundamental feature of nature, and in our view it is worth striving to eliminate loopholes in their interpretation, for reasons similar to those motivating the ongoing quest to remove Bell experiment loopholes. Although it is a theoretically familiar idea that the space and time we inhabit somehow emerge from a more fundamental quantum description, it remains an extraordinary claim, which justifies extraordinary care in assessing evidence.

We propose here extending the entanglement generated in a BMV experiment to distant quantum particles in a Bell experiment. For the reasons just given, this would provide a more definitive test of the nonclassicality of gravity and spacetime.

\section{A BELL-BOSE ET $A L$-MARLETTO-VEDRAL EXPERIMENT}

We propose an experimental test for the nonclassicality of gravity comprising three general steps. Broadly, these steps are the following (see Fig. 11).

1. Perform the BMV experiment [32, 33] with a pair of mesoscopic masses with spin. Let us call these systems "particle 1" and "particle 2", and their spin degrees of freedom " $S_{1}$ " and " $S_{2}$ ". Arrange the experiment so that the particles' states are initially unentangled, and the only non-negligible interaction between the particles during the experiment is the gravitational field. The hypothesis that gravity is quantum implies that $S_{1}$ and $S_{2}$ become entangled due to the gravitational interaction of the particles' masses [32, 33, 45]. Assuming this hypothesis is correct, arrange the experiment so that

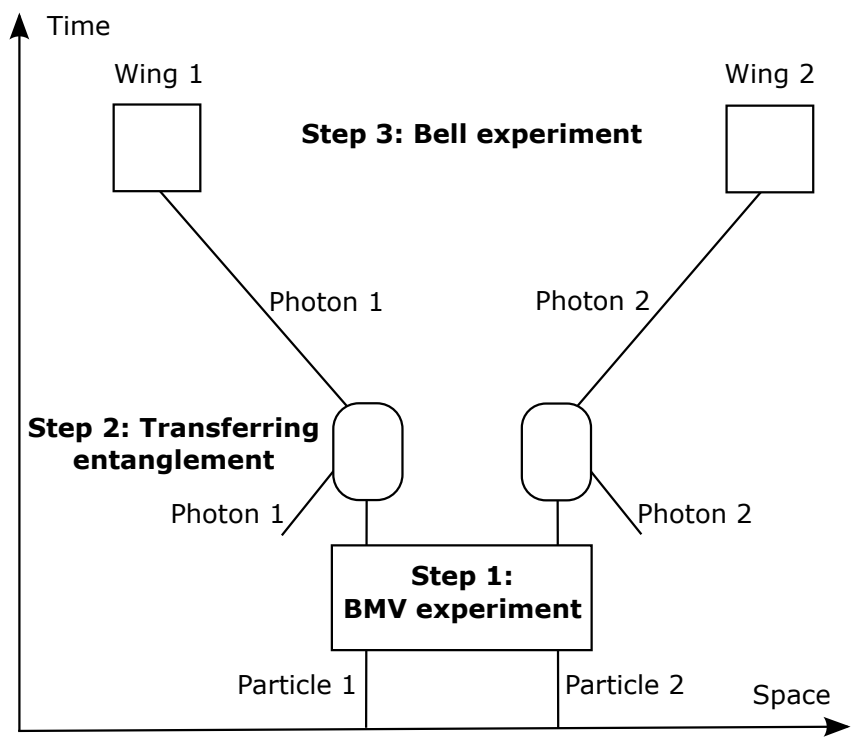

FIG. 1. Our proposed BBMV experiment. We illustrate our proposed BBMV experiment in a spacetime diagram in $1+1$ dimensions in a background Minkowski spacetime. Our proposed experiment comprises three broad steps. In step 1 (large rectangle), the BMV experiment [32, 33] is applied on Particles 1 and 2 (vertical lines). The entanglement generated via the BMV effect is transferred to Photons 1 and 2 (diagonal lines) in step 2 (rounded rectangles). In step 3 (small squares), a Bell experiment [43] is applied on Photons 1 and 2, testing (for example) CHSH inequalities [44]. If conditions 14 hold and a Bell inequality is violated then we can exclude explanations via local hidden variables, obtaining a stronger proof for the nonclassicality of the gravitational field than the original BMV experiment. The illustration is not at scale. In particular, we expect the distance between the wings in the Bell experiment to be much larger than the distance between the particles in the BMV experiment.

the final state between $S_{1}$ and $S_{2}$ is approximately the maximally entangled singlet state:

$$
\rho_{S_{1} S_{2}}^{(1)} \approx\left(\left|\Psi^{-}\right\rangle\left\langle\Psi^{-}\right|\right)_{S_{1} S_{2}}
$$

where

$$
\left|\Psi^{-}\right\rangle=\frac{1}{\sqrt{2}}(|0\rangle|1\rangle-|1\rangle|0\rangle)
$$

2. Transfer the quantum state from the spin degrees of freedom $S_{i}$ of particle $i$ to some degrees of freedom $P_{i}$ (e.g., polarization) of a photon, which we call "photon $i$ ", for $i=1,2$. Arrange the experiment so that the interaction between particle 1 (respectively 2 ) and photon 1 (2) is local, i.e., without interacting with particle 2 (1) or photon 2 (1). If at the end of step 1, the joint quantum state of the particles' spins $S_{1}$ and $S_{2}$ satisfies (2), then at the end of this step the joint quantum state of the photons' degrees of freedom $P_{1}$ and $P_{2}$ is

$$
\rho_{P_{1} P_{2}}^{(2)} \approx\left(\left|\Psi^{-}\right\rangle\left\langle\Psi^{-}\right|\right)_{P_{1} P_{2}} .
$$


3. Send photons 1 and 2 to distant wings and implement a Bell [43] experiment on systems $P_{1}$ and $P_{2}$ at spacelike separation, testing (for example) Clauser-Horne-Shimony-Holt (CHSH) [44] inequalities.

We call this a Bell-Bose et al.-Marletto-Vedral (BBMV) experiment. We emphasize the conditions that need to be satisfied experimentally.

Condition 1. At the beginning of step 1, the states of particles 1 and 2 are initially unentangled.

Condition 2. During step 1, the only non-negligible interaction between particles 1 and 2 is gravity.

Condition 3. During step 2, transferring the quantum state from the spin $S_{i}$ of particle $i$ to the degrees of freedom $P_{i}$ of photon $i$ does not include any interaction between the joint system of particle $i$ and photon $i$ with the joint system of particle $\bar{i}$ and photon $\bar{i}$, either directly or indirectly via another system, for $i=1,2$.

Condition 4. The measurements in wing $i$ in the Bell experiment of step 3 are applied on the degrees of freedom $P_{i}$ of photon $i$, for $i=1,2$.

Suppose that the experiment is repeated $N>>1$ times and that conditions 1, 4 hold in each run of the experiment. In this case, observing correlations approximately consistent with CHSH measurements on a quantum singlet, and so violating the CHSH inequality, in our BBMV experiment would imply (modulo any remaining loopholes) that the correlations obtained cannot be explained by locally causal hidden variable models [43, 44]. We see relatively little motivation for considering non-locally causal hidden variable models in the context of unifying quantum theory and general relativity, and in particular for considering such models as an explanation for correlations observed in the specific BBMV experiment described. The natural inference would thus be that the correlations are indeed generated by measurements on an approximate singlet state, and hence that the BMV sub-experiment did indeed generate entanglement.

\section{DISCUSSION}

BMV experiments involve testing for correlations that would arise from measurements on the entangled quantum states that should be generated, according to nonrelativistic quantum theory using Newtonian gravitational potentials. In principle, such correlations could also be produced by a non-quantum theory of gravity involving local hidden variables. If our proposed experiment produced results consistent with quantum theory and the BMV analyses, it would exclude the latter explanation, and hence provide stronger evidence for quantum gravity. This is not to diminish the importance of BMV's 32, 33] crucial insight. Arguably, given most theorists' Bayesian priors, our proposed experiment would further (beyond a BMV experiment) enhance their credence in quantum gravity only marginally. Still, given our incomplete understanding of nature, empirical proof is preferable to confident priors.

Ideally, all else being equal, the Bell experiment of step 3 should be as loophole free as possible. However, we see more motivation for closing some loopholes than others. There is very strong motivation for closing the locality loophole [43], since BBMV experiments are motivated by the concern that gravitational effects might be mediated by locally causal hidden variables. Ideally, the collapse locality loophole [41, 42] should also be closed, since one reason for considering this loophole is the possibility that measurement events occur only when their outcomes leave a record in the gravitational field and hence, in order to verify genuine non-locally causal correlations, we need to ensure these records are created in spacelike separated regions. Although this is more challenging, recent advances in experimental quantum information science in space (e.g., [46 51]) suggest that the collapse locality loophole could be closed in the foreseeable future [42].

It would also be worthwhile to close the freedom-ofchoice loophole [43], to eliminate any possibility that information about choices made at earlier times on one wing propagates via locally causal hidden variables (which again might be associated with the gravitational field) to influence outcomes on the other. We see less motivation for closing the detector efficiency loophole [52]; we find it harder to imagine a plausible theory in which locally causal hidden variables affect the behaviour of detectors in BBMV experiments but not in standard Bell experiments.

It is hoped that the BMV experiment could be feasible in the foreseeable future (e.g., 32, 33, 35]). Our proposed BBMV experiment appears not substantially more challenging to implement than the BMV experiment and a Bell experiment. State of the art techniques for spinphoton coupling (e.g., [53, 54] ) suggest that step 2 in our experiment could be possible in practice.

\section{ACKNOWLEDGMENTS}

The authors acknowledge financial support from the UK Quantum Communications Hub grant no. EP/T001011/1. A.K. is partially supported by Perimeter Institute for Theoretical Physics. Research at Perimeter Institute is supported by the Government of Canada through Industry Canada and by the Province of Ontario through the Ministry of Research and Innovation. A.K. thanks Fay Dowker for helpful comments. 
[1] M. Green, J. Schwarz, and E. Witten, Superstring Theory (Cambridge University Press, Cambridge, UK, 1987).

[2] C. Rovelli, Quantum Gravity (Cambridge University Press, Cambridge, UK, 2004).

[3] C. M. DeWitt and D. Rickles, The role of gravitation in physics: report from the 1957 Chapel Hill conference (epubli, Berlin, 2011) Chap. 23.

[4] K. Eppley and E. Hannah, The necessity of quantizing the gravitational field, Found. Phys. 7, 51 (1977)

[5] D. R. Terno, Inconsistency of quantum-classical dynamics, and what it implies, Found. Phys. 36, 102 (2006).

[6] C. Marletto and V. Vedral, Why we need to quantise everything, including gravity, npj Quantum Inf. 3, 29 (2017).

[7] S. Carlip, Is quantum gravity necessary?, Class. Quantum Grav. 25, 154010 (2008)

[8] N. Huggett and C. Callender, Why quantize gravity (or any other field for that matter)?, Philos. Sci. 68, S382 (2001).

[9] J. Mattingly, Why Eppley and Hannah's thought experiment fails, Phys. Rev. D 73, 064025 (2006)

[10] M. Albers, C. Kiefer, and M. Reginatto, Measurement analysis and quantum gravity, Phys. Rev. D 78, 064051 (2008).

[11] A. Kent, Simple refutation of the Eppley-Hannah argument, Class. Quantum Grav. 35, 245008 (2018).

[12] C. Møller et al., Les théories relativistes de la gravitation, Colloq. Int. CNRS 91 (1962).

[13] L. Rosenfeld, On quantization of fields, Nucl. Phys. 40, 353 (1963).

[14] T. W. B. Kibble, Relativistic models of nonlinear quantum mechanics, Commun. Math. Phys. 64, 73 (1978)

[15] T. W. B. Kibble and S. Randjbar-Daemi, Non-linear coupling of quantum theory and classical gravity, J. Phys. A: Math. Gen. 13, 141 (1980).

[16] D. N. Page and C. D. Geilker, Indirect evidence for quantum gravity, Phys. Rev. Lett. 47, 979 (1981).

[17] D. Carney, P. C. E. Stamp, and J. M. Taylor, Tabletop experiments for quantum gravity: a user's manual, Class. Quantum Grav. 36, 034001 (2019).

[18] A. Tilloy and L. Diósi, Sourcing semiclassical gravity from spontaneously localized quantum matter, Phys. Rev. D 93, 024026 (2016).

[19] D. E. Bruschi and F. K. Wilhelm, Self gravity affects quantum states, arXiv:2006.11768.

[20] A. Kent, Testing quantum gravity near measurement events, Phys. Rev. D 103, 064038 (2021)

[21] A. Kent, Quantum state readout, collapses, probes, and signals, Phys. Rev. D 103, 064061 (2021).

[22] F. Karolyhazy, Gravitation and quantum mechanics of macroscopic objects, Nuovo Cimento A (1965-1970) 42, 390 (1966).

[23] L. Diósi, Gravitation and quantum-mechanical localization of macro-objects, Phys. Lett. 105A, 199 (1984).

[24] L. Diósi, A universal master equation for the gravitational violation of quantum mechanics, Phys. Lett. 120A, 377 (1987)

[25] L. Diósi, Models for universal reduction of macroscopic quantum fluctuations, Phys. Rev. A 40, 1165 (1989).

[26] R. Penrose, On gravity's role in quantum state reduction, Gen. Relat. Gravit. 28, 581 (1996)
[27] R. Penrose, Quantum computation, entanglement and state reduction, Phil. Trans. R. Soc. A. 356, 1927 (1998).

[28] R. Penrose, On the gravitization of quantum mechanics 1: quantum state reduction, Found. Phys. 44, 557 (2014).

[29] R. Howl, R. Penrose, and I. Fuentes, Exploring the unification of quantum theory and general relativity with a Bose-Einstein condensate, New J. Phys. 21, 043047 (2019).

[30] S. Donadi, K. Piscicchia, C. Curceanu, L. Diósi, M. Laubenstein, and A. Bassi, Underground test of gravity-related wave function collapse, Nat. Phys. 17, 74 (2021).

[31] S. Weinstein and D. Rickles, Quantum Gravity, in The Stanford Encyclopedia of Philosophy, edited by E. N. Zalta (Metaphysics Research Lab, Stanford University, Stanford, California, 2021) Fall 2021 ed.

[32] S. Bose, A. Mazumdar, G. W. Morley, H. Ulbricht, M. Toroš, M. Paternostro, A. A. Geraci, P. F. Barker, M. S. Kim, and G. Milburn, Spin entanglement witness for quantum gravity, Phys. Rev. Lett. 119, 240401 (2017)

[33] C. Marletto and V. Vedral, Gravitationally induced entanglement between two massive particles is sufficient evidence of quantum effects in gravity, Phys. Rev. Lett. 119, 240402 (2017)

[34] There are other possibilities. For example, one can imagine theories in which gravity is mediated by quantum particles but spacetime superpositions are precluded or in which the fundamental theory of spacetime is not described by a quantum formalism.

[35] T. W. van de Kamp, R. J. Marshman, S. Bose, and A. Mazumdar, Quantum gravity witness via entanglement of masses: Casimir screening, Phys. Rev. A 102, 062807 (2020).

[36] S. A. Haine, Searching for signatures of quantum gravity in quantum gases, New J. Phys. 23, 033020 (2021)

[37] R. Howl, V. Vedral, D. Naik, M. Christodoulou, C. Rovelli, and A. Iyer, Non-gaussianity as a signature of a quantum theory of gravity, PRX Quantum 2, 010325 (2021).

[38] D. Carney, H. Müller, and J. M. Taylor, Using an atom interferometer to infer gravitational entanglement generation, PRX Quantum 2, 030330 (2021)

[39] M. J. W. Hall and M. Reginatto, On two recent proposals for witnessing nonclassical gravity, J. Phys. A: Math. Theor. 51, 085303 (2018).

[40] C. Marletto and V. Vedral, Witnessing nonclassicality beyond quantum theory, Phys. Rev. D 102, 086012 (2020).

[41] A. Kent, Causal quantum theory and the collapse locality loophole, Phys. Rev. A 72, 012107 (2005).

[42] A. Kent, Stronger tests of the collapse-locality loophole in Bell experiments, Phys. Rev. A 101, 012102 (2020).

[43] J. S. Bell, On the Einstein Podolsky Rosen paradox, Physics 1, 195 (1964), reprinted in [55], pages 14-21.

[44] J. F. Clauser, M. A. Horne, A. Shimony, and R. A. Holt, Proposed experiment to test local hidden-variable theories, Phys. Rev. Lett. 23, 880 (1969).

[45] M. Christodoulou and C. Rovelli, On the possibility of laboratory evidence for quantum superposition of geometries, Phys. Lett. B 792, 64 (2019)

[46] J. Yin et al., Satellite-based entanglement distribution 
over 1200 kilometers, Science 356, 1140 (2017)

[47] S.-K. Liao et al., Satellite-to-ground quantum key distribution, Nature (London) 549, 43 (2017).

[48] S. K. Joshi et al., Space QUEST mission proposal: experimentally testing decoherence due to gravity, New J. Phys. 20, 063016 (2018).

[49] J. S. Sidhu et al., Advances in space quantum communications, IET Quantum Commun. 2, 182 (2021)

[50] R. Kaltenbaek et al., Quantum technologies in space, Exp. Astron. 51, 1677 (2021).

[51] G. Gasbarri, A. Belenchia, M. Carlesso, S. Donadi, A. Bassi, R. Kaltenbaek, M. Paternostro, and H. Ulbricht, Testing the foundation of quantum physics in space via interferometric and non- interferometric experiments with mesoscopic nanoparticles, Commun. Phys. 4, 155 (2021).

[52] P. M. Pearle, Hidden-variable example based upon data rejection, Phys. Rev. D 2, 1418 (1970)

[53] X. Mi, M. Benito, S. Putz, D. M. Zajac, J. M. Taylor, G. Burkard, and J. R. Petta, A coherent spin-photon interface in silicon, Nature (London) 555, 599 (2018).

[54] N. Samkharadze, G. Zheng, N. Kalhor, D. Brousse, A. Sammak, U. C. Mendes, A. Blais, G. Scappucci, and L. M. K. Vandersypen, Strong spin-photon coupling in silicon, Science 359, 1123 (2018)

[55] J. S. Bell, Speakable and unspeakable in quantum mechanics (Cambridge University Press, Cambridge, 1987). 